\title{
CLASSIFICATION OF BACTERIAL IMAGES USING TRANSFER LEARNING, OPTIMIZED TRAINING AND RESNET-50
}

\author{
Daniel Martomanggolo Wonohadidjojo
}

Ciputra University Surabaya, Indonesia

Email: daniel.m.w@ciputra.ac.id

\begin{tabular}{|c|c|}
\hline ART & ABSTRACT \\
\hline $\begin{array}{l}\text { Received: } \\
\text { January, } 26^{\text {th }} \\
2022 \\
\text { Revised: } \\
\text { February, } 17^{\text {th }} \\
2022 \\
\text { Approved: } \\
\text { February, } 18^{\text {th }} \\
2022\end{array}$ & $\begin{array}{l}\text { Bacterial image analysis using traditional laboratory } \\
\text { methods encounters bacterial recognition errors and } \\
\text { requires extra experience and long processing time. } \\
\text { Therefore, the automated classification technique of } \\
\text { bacterial images is more useful than traditional visual } \\
\text { observations for biologists because of their accurate } \\
\text { classification, low cost, and fast diagnosis. In this study, a } \\
\text { method to classify bacteria images by implementing the } \\
\text { CNN deep learning method using Transfer Learning is } \\
\text { proposed. This trained ResNet-50 is implemented as the } \\
\text { CNN architecture. In the training of the classification layer, } \\
\text { SGDM optimizer is used. The classification performance for } \\
\text { is evaluated in using confusion matrix and four } \\
\text { performance metrics: Accuracy, Precision, Recall and } \\
\text { Fmeasure. The Confusion Matrix and all the performance } \\
\text { metrics show is successful in classifying bacterial images. }\end{array}$ \\
\hline KEYWORDS & $\begin{array}{l}\text { Bacteria classification, Convolutional Neural Network, } \\
\text { ResNet-50, Transfer Learning. }\end{array}$ \\
\hline cc) (†) & $\begin{array}{l}\text { his work is licensed under a Creative Co } \\
\text { ttribution-ShareAlike } 4.0 \text { International }\end{array}$ \\
\hline
\end{tabular}

\section{INTRODUCTION}

The recognition of bacterial samples carried out in culturing process requires equipment and chemical substance used for staining the samples. Moreover, rigorous culturing procedures and safety protocols must be met. After that the results are obtained,

\begin{tabular}{cl}
\hline & Daniel Martomanggolo Wonohadidjojo. (2022). Classification of \\
& Bacterial Images Using Transfer Learning, Optimized Training and \\
How to cite: & Resnet-50. Journal Eduvest. Vol 2(2): 297-305 \\
E-ISSN: & 2775-3727 \\
Published by: & https://greenpublisher.id/
\end{tabular}




\section{Daniel Martomanggolo Wonohadidjojo}

the samples need to be analysed to find characteristic features and to classify the particular genera and species of bacteria. Using this classical method in the laboratory, the bacteria recognition requires knowledge and experience of experts. The problem encountered using this method are the time-consuming process and the requirement of comparative analysis of the obtained samples with the reference ones (Zieliński et al., 2017).

Biologists identify and classify types of bacteria based on different biochemicals and forms. These are the bacterial attributes which are used for classification. The shape of cell, the size and structure of the colonies formed by the bacteria are used to distinguish bacterial species. The bacterial cell can be spiral, cylindrical, and spherical. Cells of several types of bacteria have different sizes and structures depending on environmental conditions (Handoyo \& Mulyandari, 2021). Several species of bacteria have very similar shapes. Each bacterial species has its own characteristics, the biochemical reactions and the metabolic activities carried out by the bacteria help to classify the species. However, the task to classify bacterial species is not easy even for an experienced specialist (Talo, 2019).

In general, microbiological image analysis using traditional laboratory methods encounters bacterial recognition errors and requires extra experience and long processing time. Therefore, the automated classification technique of bacterial images is more useful than traditional visual observations for biologists because of its accurate classification, low cost, and fast diagnosis. Several studies related to bacterial classification have been conducted and several methods have been proposed. In 2018 a classification method using CNN with the AlexNET architecture was proposed. It was used to classify 18 categories of bacteria with an accuracy of $73 \%$ (Huang \& Wu, 2018).

Then in 2019 another study to classify two types of bacteria using deep learning method with LeNET architecture was conducted with more than $75 \%$ accuracy of the results (Treebupachatsakul \& Poomrittigul, 2019). Deep learning increases its remarkable position in computer vision when neural systems began to outperform other strategies on a few high profile image analysis benchmarks (Lundervold \& Lundervold, 2019). The CNN methods have been found to either outperform or complement the existing traditional and Machine Learning approaches in terms of accuracy, sensitivity, Area Under Curve, Dice Similarity Coefficient, and time taken (Sarvamangala \& Kulkarni, 2021).

In this study, a method to classify bacteria by implementing the CNN deep learning method using Resnet-50 architecture is proposed. The ResNet-50 architecture is implemented in this study with the consideration of its performance. Previous studies shows its performance in classifying digital images with high accuracy results such as the classification of brain tumors (Mohsen, El-Dahshan, El-Horbaty, \& Salem, 2018), malaria infected cells (Sai Bharadwaj Reddy \& Sujitha Juliet, 2019), and protein cells (Elnashar, Abd, \& Azim, 2021). Furthermore, in this study the training process in Transfer Learning is optimized using Stochastic Gradient Descent with Momentum (SGDM) algorithm. The use of SGDM in machine learning task has been verified in several studies. A stochastic optimization algorithm performs better in terms of expected error, and hence, makes a better learning algorithm (Bottou, Curtis, \& Nocedal, 2018). 


\section{RESEARCH METHOD}

The trained network used in this transfer learning is the CNN model. CNN is a type of neural network which uses special technique called convolution. CNN has 2 major layers, namely the layer for feature extraction and the layer for classification. Layers for feature extraction consist of convolutional layers, pooling layers, stride, and padding. Meanwhile, the layers for classification consists of a fully connected layer, softmax, and an output layer.

ResNet-50 is an architecture from CNN which introduces a new concept, namely shortcut connections. The emergence of the concept of shortcut connections in the ResNet-50 architecture is related to the vanishing gradient problem that occurs when efforts to deepen the structure of a network are carried out. However, deepening a network with the aim of improving its performance cannot be done simply by stacking layers. The deeper a network can lead to a vanishing gradient problem that can make the gradient very small which results in decreased performance or accuracy.

ResNet introduces the concept of shortcut connections and in this concept features which are inputs from the previous layer are also used as inputs to the outputs of that layer. This method is implemented as a solution to minimize the loss of important features during the convolution process.

This trained network is used in the transfer learning method in this study. The transfer learning stage used in this study includes importing a previously trained network, replacing the classification layer, training the network on the new dataset whish is the bacteria cell images, classifying the bacteria and getting accurate performance measures. Figure 1 shows the transfer learning method used in this study.

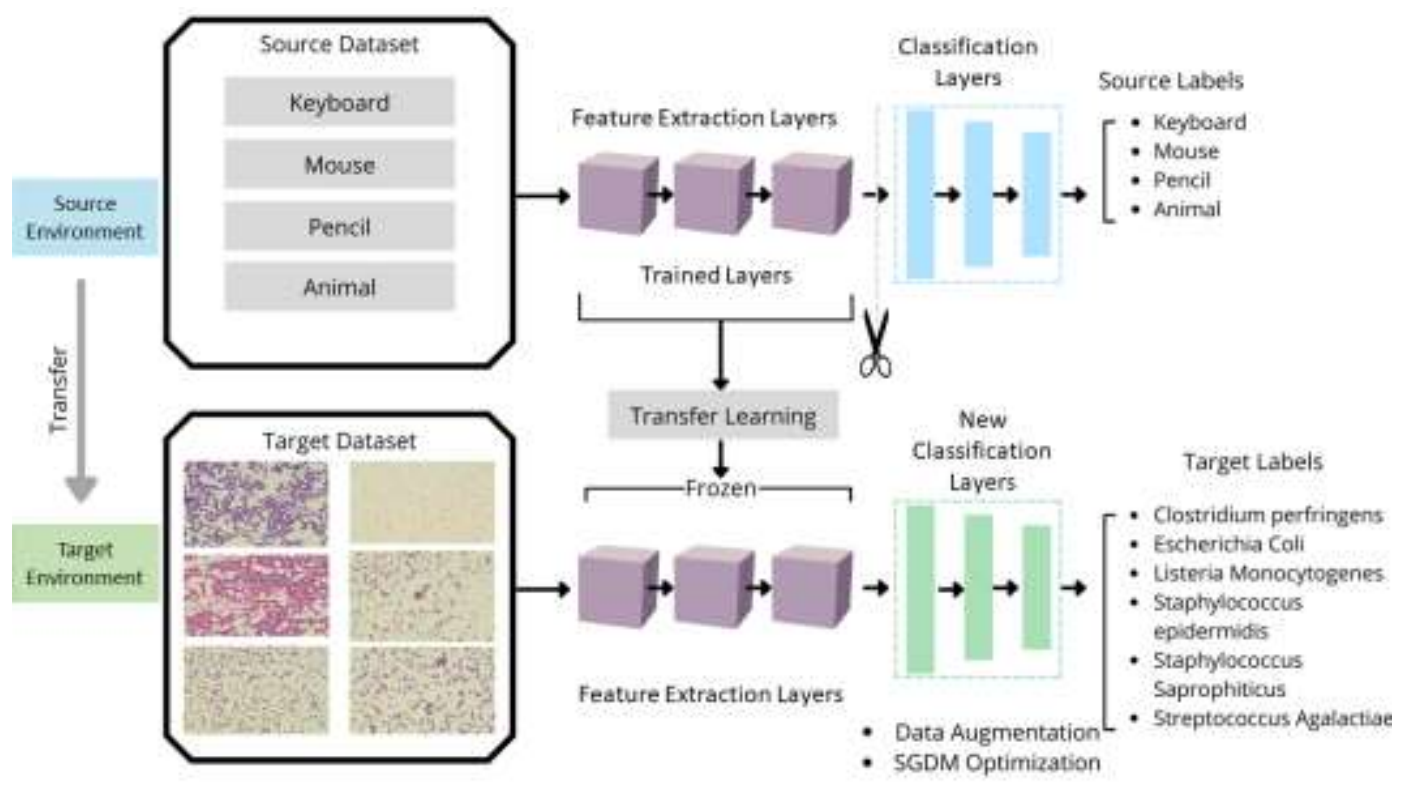

Figure 1. The Transfer Learning Method. 


\section{A. Importing the Trained Network}

In this study, the network architecture used is ResNet-50 as the network that has been trained. The ResNet-50 architecture is a Residual Network consisting of 50 layers. The layers at the beginning and the middle are called feature extraction layer. These layers process a range of features from simple ones such as brightness and edges to uniquely complex ones such as color and shape. The learning outcomes of the feature extraction layer in the source environment (the environment where the network originates) can be transferred to the feature extraction layers in the target environment (the new environment in this study). Feature extraction learning in the target environment is used to extract the bacterial image data used in the training phase.

\section{B. Transfer learning}

In this study, transfer learning method is used for network learning that utilizes the classification layers. The classification layers are known as the last 3 layers, namely fully connected, softmax, and output layers. The original classification layers are replaced with new classification layers that are suitable for the new classification task. These new layers also include the number of new classes and the learning speed set in the new network.

The classification layers on the original network are configured for the number of classes in the previous training. These layers in the network architecture contains information that is processed by the network into class probabilities, loss values, and predicted labels. To classify a new image, these layers are replaced with new layers and adjusted to the new dataset. The fully connected layer is replaced with a new layer that has the same number of outputs as the number of classes. To make learning process in the new layer faster compared to the previous layer, the learning speed factor is increased. The classification layers determine the network output class. The classification layers are replaced with the new ones without the class label. During training, the network automatically determines the output class of that layers.

In the input of convolutional layer, the network requires an input image of a certain size, but the input images have different sizes. The image size is adjusted automatically such that it can be used for the training network. Furthermore, data augmentation operations is carried out on the training dataset by applying random affine geometric transformations, namely horizontal reflection technique, and horizontal and vertical translation technique. Data augmentation helps prevent the network from overfitting and remembers precise details from training images.

In the training process, the learning speed parameters are determined for transfer learning by maintaining the features in the initial network layer that have been trained previously (the weight of the transferred layer). The learning rate in the transferred layer needs to be decreased, so the initial learning speed is set to a small value. The learning rate in the new classification layer needs to be accelerated, so the learning speed factor in the previous step has been increased. The combination of these learning speed setting results in fast learning only in the new layer and slower learning in the other layers.

While doing transfer learning, there is no need for training with the same number of epochs as the previous number. An epoch is a complete training cycle across the entire training dataset. In this training, the mini batch file size and validation data are also determined. Network validation is carried out every iteration with a predetermined frequency during training. Furthermore, training is carried out on the network consisting 
of the transferred layer and the new layer using the GPU. Then the image classification is undertaken using a network that has gone through the training.

C. Optimization of the network training

In this study we apply optimization in the training process of Transfer Learning.

The Gradient Descent (GD) optimization method is applied with learning rate value of 0.0001. GD will update weights after all training data has been processed, or every 1 epoch (iteration). Because the learning rate is small, to avoid the long process to reach the optimum state, the Stochastic Gradient Descent (SGD) solution is implemented. SGD updates the weight without waiting for an epoch to finish. SGD uses a concept similar to batching by dividing the training data into several batches. The weight will be updated in each batch after processing. To further improve the performance, a momentum weight of $\beta>0$ is applied so that the optimization algorithm used in this study is Stochastic Gradient Descent with Momentum (SGDM).

\section{Dataset and experiment}

The dataset for training is bacteria images which is publicly available at http://misztal.edu.pl/software/databases/dibas/. In this study the dataset used consists of five types of bacteria that commonly cause infection in humans and one type of bacteria that causes infection in animal. The type of bacteria that causes infection in animal is also used as dataset to prove that the proposed method in this study is also able to classify them among other bacteria that cause infections in humans. The training used 125 digital bacteria images consisting of 6 classes, namely 23 Clostridium perfringens, 20 Escherichia Coli, 22 Listeria Monocytogenes, 20 Staphylococcus epidermidis, 20 Staphylococcus Saprophiticus and 20 Streptococcus Agalactiae. Clostridium perfringens bacteria are the type of bacteria of the most common causes of foodborne illness (food poisoning). Escherichia coli (E. coli) bacteria are bacteria that live in the human intestine to maintain a healthy digestive system. Listeria monocytogenes is the species of pathogenic bacteria that can cause serious and fatal infections in infants, children, the sick and the elderly, and people with weakened immune systems. Staphylococcus epidermidis is a species of bacteria from the genus Staphylococcus which is known to cause opportunistic infections (attacking individuals with weak immune systems). Staphylococcus Saprophiticus can cause urinary tract infections in young women. Streptococcus agalactiae is a species of bacteria that causes streptococcosis in Nile tilapia (Oreochromis niloticus).

With 125 images in dataset for training, the training process can take place faster than using more images in dataset. With this number of dataset, fine-tuning the new network can still work better, because the new network has learned the new features as the result of previous training. This is an advantage of the transfer learning method used in this study.

The size of each digital image is pixels. The experimental setup is as follows: the dataset is divided into two parts, $70 \%$ for training and $30 \%$ for testing. The size of all images is $2048 \times 1532$ pixels. For the purposes of this training where image variations are required, dataset augmentation is also implemented. Figure 2 shows examples of Clostridium perfringens, Escherichia Coli, Listeria Monocytogenes, Staphylococcus epidermidis, Staphylococcus Saprophiticus and Streptococcus Agalactiae images respectively. 


\section{Daniel Martomanggolo Wonohadidjojo}

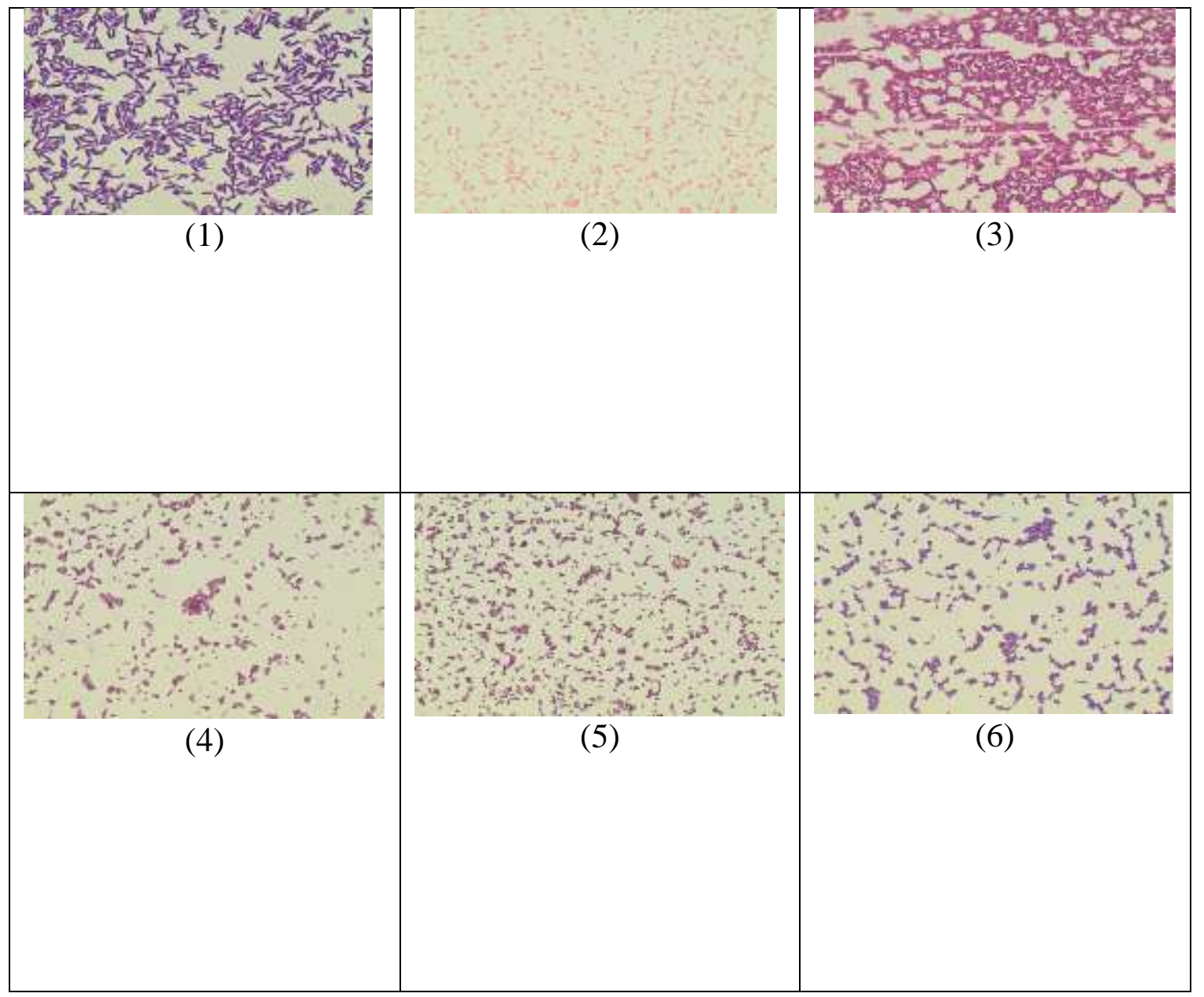

Figure 2. Examples of Bacteria Digital Images: (1) Clostridium perfringens (2) Escherichia coli (3) Listeria monocytogenes (4) Staphylococcus epidermidis (5) Staphylococcus saprophyticus (6) Streptococcus agalactiae

The training parameters used for all networks are as follows: learning rate 1e-4, minibatch size 10, max epoch 30 , validation frequency 3 .

The network architecture is implemented with MATLAB on an Intel(R) Core(TM) i7-8565U CPU @ 1.80GHz 1.99 GHz and 16.0 GB RAM with 1 GB NVIDIA graphics processing unit (GPU).

\section{RESULT AND DISCUSSION}

In the experiments that have been carried out, all network architectures have succeeded in classifying bacteria images using transfer learning. An example of bacteria classification results is shown in Figure 3. 


\section{A. Confusion matrix}

The classification performance for each network architecture is presented in the form of a confusion matrix as shown in Figure 3. To evaluate the results of the classification, four performance metrics are used, namely Accuracy, Precision, Recall and Fmeasure. The results of the performance metrics measurements are presented in Table 1.

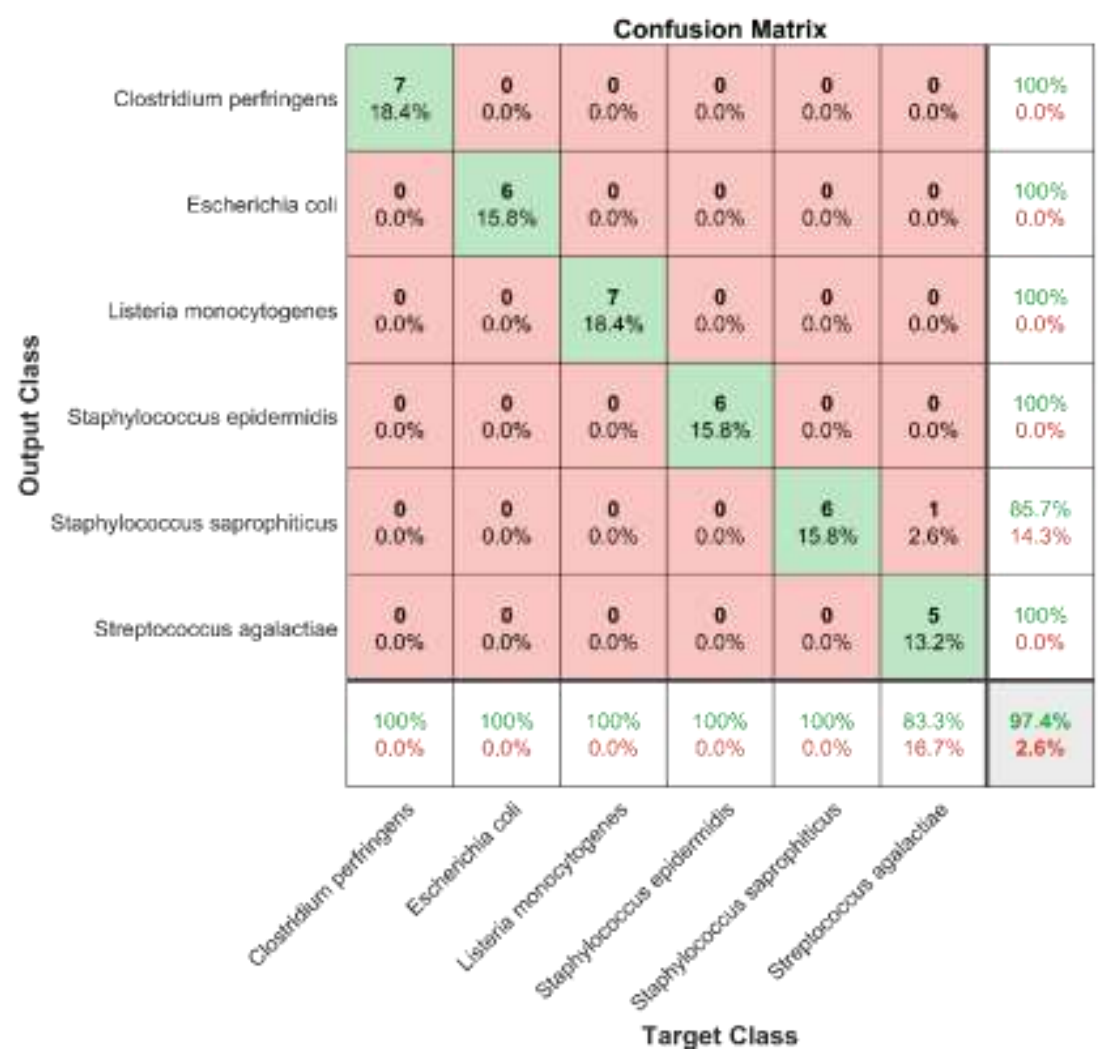

Figure 3. Confusion Matrix of the Proposed Method.

Table 1. Performance Metrics of the Proposed Method

\begin{tabular}{cccc}
\hline Accuracy & Precision & Recall & Fscore \\
\hline $97.37 \%$ & 0.9761 & 0.9722 & 0.9742 \\
\hline
\end{tabular}

Values on the main diagonal of CM represent all correctly classified examples. The row below each CM shows the level of accuracy achieved for each predicted class and the class prediction error. The CM shows that almost all images are classified correctly, only 1 image classified incorrectly. This shows that the proposed method is successful in the classification task.

B. Evaluation using Performance metrics

Table 1 shows performance metrics of the proposed method measured using four metrics: Accuracy, Precision, Recall and Fscore. All the metrics show consistent values that verify the result of Confusion Matrix. This shows that the proposed method is successful in classifying the bacteria images. 


\section{Daniel Martomanggolo Wonohadidjojo}

\section{CONCLUSION}

In this study, a method to classify bacterial images using transfer learning has been proposed. In this method, transfer learning using ResNet-50 network architecture that has been trained beforehand is applied to classify the images. In the new environment the training process is optimized using SGDM algorithm. To increase the number and variation of dataset and to avoid overfitting problem, data augmentation is implemented. To evaluate the performance of the network architecture, CM and four performance metrics namely Accuracy, Precision, Recall and Fmeasure are used.

The results of this study show that almost all the images are classified correctly, with only one image classified incorrectly. The performance metrics show consistent result where accuracy is $97.37 \%$, precision is 0.9761 , recall is 0.9722 and Fscore is 0,9742 . These results show that the proposed method is successful in classifying bacteria images. They suggest that the proposed method offers a new method to be used in other target environment to classify bacteria for medical or other same kind of purposes.

For future research direction, it is recommended to increase the values of performance metrics by applying other optimization technique or algorithm.

\section{REFERENCES}

Bottou, L., Curtis, F. E., \& Nocedal, J. (2018). Optimization methods for large-scale machine learning. SIAM Review, 60(2), 223-311. https://doi.org/10.1137/16M1080173

Elnashar, H., Abd, I., \& Azim, E. (2021). Deep Learning : Protein Cells Classifications using Resnet-50 Model. 10(06), 849-855.

Handoyo, S., \& Mulyandari, E. (2021). Analisis Imbangan Air pada Daerah Irigasi Jetu Kabupaten Karanganyar. Syntax Literate; Jurnal Ilmiah Indonesia, 6(8), 40934106.

Huang, L., \& Wu, T. (2018). Novel neural network application for bacterial colony classification. Theoretical Biology and Medical Modelling, 15(1), 1-16. https://doi.org/10.1186/s12976-018-0093-x

Lundervold, A. S., \& Lundervold, A. (2019). An overview of deep learning in medical imaging focusing on MRI. Zeitschrift Fur Medizinische Physik, 29(2), 102-127. https://doi.org/10.1016/j.zemedi.2018.11.002

Mohsen, H., El-Dahshan, E.-S. A., El-Horbaty, E.-S. M., \& Salem, A.-B. M. (2018). Classification using deep learning neural networks for brain tumors. Future Computing and Informatics Journal, 3(1), 68-71. https://doi.org/10.1016/j.fcij.2017.12.001

Sai Bharadwaj Reddy, A., \& Sujitha Juliet, D. (2019). Transfer learning with RESNET50 for malaria cell-image classification. Proceedings of the 2019 IEEE International Conference on Communication and Signal Processing, ICCSP 2019, 945-949. https://doi.org/10.1109/ICCSP.2019.8697909

Sarvamangala, D. R., \& Kulkarni, R. V. (2021). Convolutional neural networks in medical image understanding: a survey. Evolutionary Intelligence, (0123456789). https://doi.org/10.1007/s12065-020-00540-3

Talo, M. (2019). An Automated Deep Learning Approach for Bacterial Image Classification. 1-5.

Treebupachatsakul, T., \& Poomrittigul, S. (2019). Bacteria Classification using Image 
Processing and Deep learning. 34th International Technical Conference on Circuits/Systems, Computers and Communications, ITC-CSCC 2019, 2-4. https://doi.org/10.1109/ITC-CSCC.2019.8793320

Zieliński, B., Plichta, A., Misztal, K., Spurek, P., Brzychczy-Włoch, M., \& Ochońska, D. (2017). Deep learning approach to bacterial colony classification. PLoS ONE, 12(9). https://doi.org/10.1371/journal.pone.0184554 